晅吧枵上

$\mathrm{Vol} 7 \mathrm{~N}^{\circ} 2202 \mathrm{O}$
REVUE

DE LA SOCIÉTÉ

DE PHILOSOPHIE

DES SCIENCES

http://dx.doi.org/10.20416/LSRSPS.V7I2.1

\title{
Juliette Ferry-Danini
}

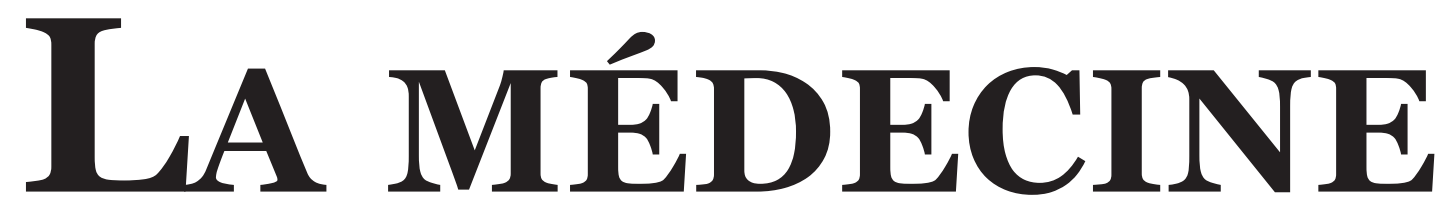

NARRATIVE FACE
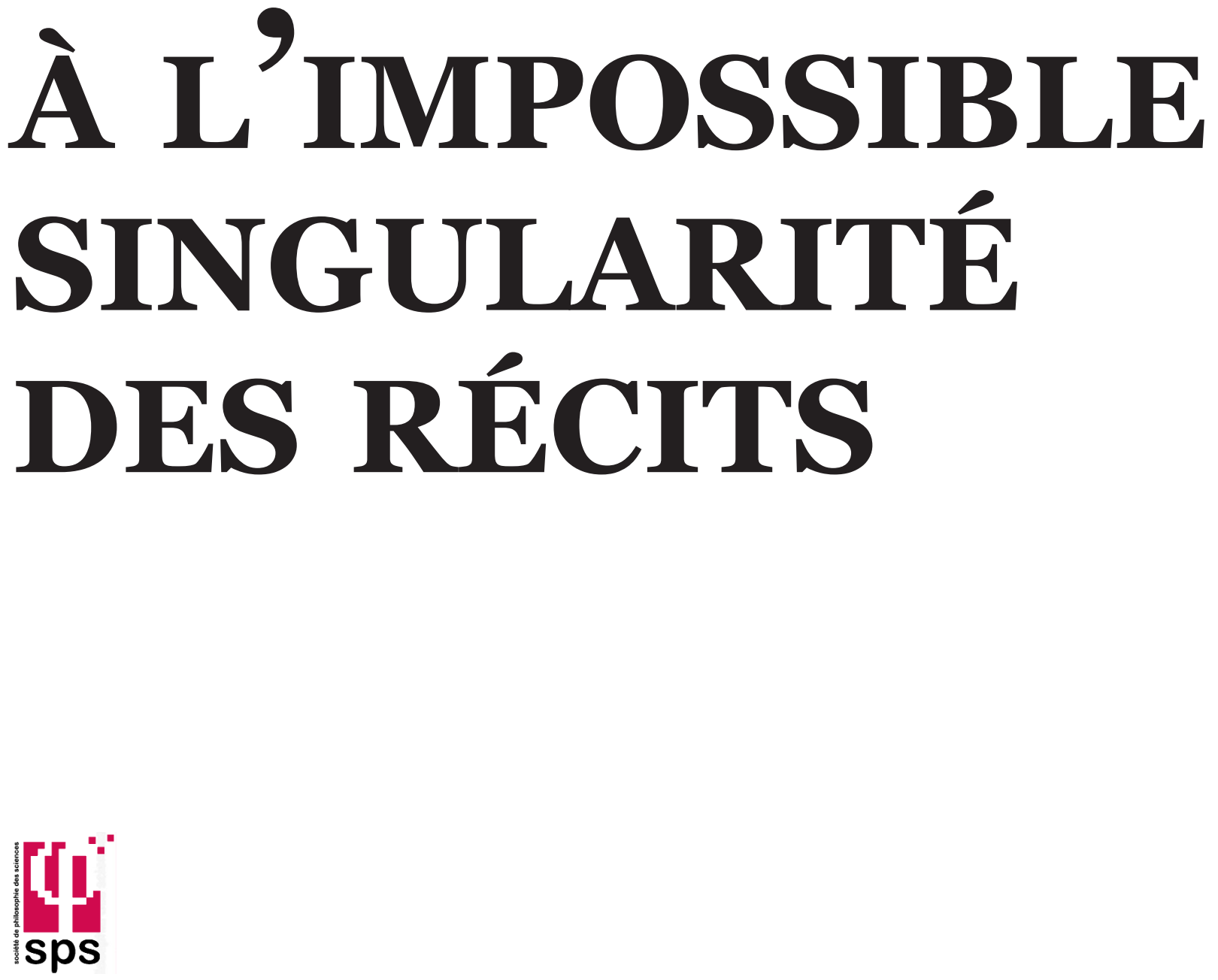

SOCIÉTÉ DE PHILOSOPHIE DES SCIENCES (SPS) 


\section{LA MÉDECINE NARRATIVE FACE À L'IMPOSSIBLE SINGULARITÉ DES RÉCITS ${ }^{1}$}

\section{Sommaire \\ 1 - Introduction \\ 2 - La thèse de la singula- rité des récits des patients au cour de la médecine narra- \\ tive \\ 3 - La Recherche n'illustre-t- elle qu'elle-même? 4- Quand des types de récits de maladie deviennent domi- nants : l'exemple du cancer $d u$ sein aux États-Unis au XXe siècle 5 - Conclusion}

\section{1 - Introduction}

« Un nouveau patient est comme une nouvelle planète » Rita Charon, pendant sa conférence plénière lors du colloque « Médecine \& Récit. La médecine comme expérience biographique, » 19 octobre 2017 à Créteil, France ${ }^{2}$.

La médecine narrative (Brody, 1997 ; Charon, 2001 ; 2006 ; Goupy \& Jeune, 2016 ; Frank, 1997 ; Hunter, 1991 ; Vannatta \& Vannatta 2013) a récemment gagné en popularité et en visibilité dans la médecine ${ }^{3}$ et dans la philosophie de la médecine (voir les articles et ouvrages critiques sur le sujet, Reiss, Solomon, \& Teira 2011; Solomon, 2008; 2015; Woods, 2011; Ferry-Danini, 2018). L'expression « médecine narrative » est brandie comme un slogan pour défendre l'idée que la narration et la théorie littéraire sont essentielles pour la médecine et sa pratique. Au-delà des tribunes générales pour l'intégration d'enseignements littéraires dans le cursus de médecine, la médecine narrative défend des thèses philosophiques sur la pratique médicale et son rapport au récit. C'est cette dernière dimension qui m'intéresse ici. L'une des thèses philosophiques fondatrices de la médecine narrative soutient que l'expérience de la maladie étant absolument unique et singulière ${ }^{4}$, sa compréhension échappe au domaine de la science et peut seulement être abordée via la théorie littéraire et narrative. Reformulée comme " médecine narrative ", seul cet « art » permettrait de toucher au caractère singulier des récits des expériences de la maladie racontés par les patients. Dans cet article, je souligne quelques limites de cette thèse.

\section{2 - La thèse de la singularité des récits des patients au cour de la médecine narrative}

Formulons d'abord les principales thèses défendues par la médecine narrative, notamment dans la version portée par Rita Charon, l'une des figures de proue du mouvement. Deux thèses principales caractérisent la médecine narrative. Selon la plus radicale de ces deux thèses, des compétences dites " narratives » sont essentielles à une bonne pratique de la médecine. Rita Charon écrit : promouvoir une "nouvelle philosophie de la connaissance médicale » fondée sur les théories narratives, qui permet d'améliorer « l'efficacité du soin » (Charon, 2006, p. 39). Un bon lecteur ferait un bon médecin, autrement dit, la connaissance des théories littéraires - la narratologie - et la maîtrise de ses outils, permettraient une médecine plus efficace. Vannatta et Vannatta soutiennent que «l'éducation des médecins aux compétences narratives devrait permettre de meilleurs diagnostics grâce à une meilleure compréhension du récit du patient » (2013, p. 35). Que désignent ces compétences narratives ? Rita Charon fait référence à la narratologie et notamment aux travaux de plusieurs théoriciens et théoriciennes, entre autres, Gérard Genette, Tzvetan Todorov et Shlomith Rimmon-Kenan. Par exemple, Charon écrit que « le sens est appréhendé de façon collaborative, par le lecteur et l'écrivain, l'observateur et celui qui est observé, le médecin et le patient » (Charon, 2001, p. 1898). Une maitrise des théories de la lecture, selon elle, permettrait de mieux cerner la relation entre le patient et son médecin. Kathryn M. Hunter compare quant à elle le travail du médecin à la figure de Sherlock Holmes (Hunter, 1991, p. 24), au sens où le médecin doit écouter un récit, le démêler,

1 - Cette publication remplace la version précédente de cet article, publiée en ligne sur la plateforme HAL sous le titre « La singularité des récits des patients: un écueil pour la médecine narrative?".

2 - Traductions personnelles.

3 - Il existe des programmes de médecine narrative dans plusieurs universités américaines. Par exemple à l'université de médecine de Columbia. Des formations similaires commencent à apparaitre en France, par exemple lïnitiative de formation en cinq jours à la médecine narrative proposée par la Faculté de médecine de Nice, du 28 février au 3 mars 2018. En France, des colloques sur le sujet sont périodiquement organisés en vue de présenter la méthode aux professionnels de la santé (voir par exemple le colloque « Médecine et Récit. La maladie comme expérience biographique » en octobre 2017 à Créteil et Paris).

4-Ces mots seront utilisés de façon synonyme dans le reste de l'article. 


\section{LA MÉDECINE NARRATIVE FACE À L'IMPOSSIBLE SINGULARITÉ DES RÉCITS}

en extraire des informations et faire des déductions.

Selon une thèse sensiblement moins polémique, mais significativement plus répandue au sein de la médecine narrative et ailleurs, l'attention du médecin aux récits des patients est une approche privilégiée pour prendre en compte les besoins psychologiques et émotionnels de ceuxci. L'idée, très commune dans les humanités médicales, est que l'écoute attentive du récit du patient permet de redonner du sens à la pratique de la médecine, voire de l'humaniser et de compléter ainsi la biomédecine. Comme l'écrit Charon, il s'agit d' "imprégner les faits et les objets de la santé et la maladie de leurs conséquences et du sens qu'ils ont pour les patients et leurs médecins » (Charon, 2001, p. 1898). Cette thèse - la médecine narrative comme moyen d'humaniser la médecine - repose sur une série de thèses philosophiques : 1) l'expérience de chaque patient est absolument unique ( « singulière » pour reprendre le vocabulaire de Charon) ; 2) le récit de cette expérience consiste en cette expérience (ce qui correspond à la thèse de l'identité narrative), ou du moins restitue fidèlement cette expérience ; 3) chaque récit de maladie est donc aussi absolument unique ; 4) dans la rencontre clinique, l'histoire du patient doit être prise en compte dans sa singularité ; 5) seule une approche narrative peut prendre en compte l'expérience singulière et unique d'un patient.

Ces thèses sont autant de prémisses qui permettent aux défenseurs de la médecine narrative de conclure que la meilleure médecine possible - une médecine humaine ou humaniste - doit être narrative. Cependant, ni ces prémisses ni cet argument ne vont de soi. D'autres auteurs ont déjà remarqué que la thèse de l'identité narrative (l'identité entre les expériences des patients et leurs récits) est un postulat qui n'est pas défendu par les défenseurs de la médecine narrative (O'Mahony, 2013). Charon se contente notamment de citer Todorov et l'idée selon laquelle le récit ne rapporte pas l'expérience qui lui préexisterait, mais qu'elle la produit véritablement (Charon, 2006, p. 45). Laissons cette objection de côté, et admettons que raconter une histoire permette de rendre compte plus ou moins fidèlement de son expérience ${ }^{5}$; des obstacles demeurent néanmoins. Notamment, est-ce vraiment pertinent d'affirmer que chaque récit de patient est unique ? Peut-on dire que l'étude littéraire permet d'accéder à l'expérience d'un individu dans sa singularité absolue ? Enfin, la singularité des récits des patients est-elle un bon argument en faveur de la médecine narrative ? Dans ce qui suit, je montrerai que ces thèses ne vont pas de soi et que paradoxalement elles vont parfois à l'encontre des racines théoriques de la médecine narrative.

\section{3 - La Recherche n'illustre-t- elle qu'elle-même?}

Plusieurs défenseurs de la médecine narrative ont souligné le lien entre la médecine narrative, l'intérêt pour les récits des patients, et la possibilité de prendre en compte la singularité des expériences des patients par ce moyen. Par exemple, M. K. Hunter soulignait déjà l'unicité de l'expérience des patients, en remarquant que si

« Les cliniciens et les chercheurs cliniciens travaillent sur la cartographie de la maladie (...) chaque patient et chaque cas de maladie sont des territoires inexplorés. » (Hunter, 1991, p. 14) ${ }^{6}$

Dans une conférence donnée en octobre 2017 à Créteil, Charon a d'ailleurs fait écho à cette formule, en déclarant que " chaque patient est comme une nouvelle planète ». C'est rapidement qu'elle aborde dans son livre de 2006, dès la page 45, la question de la singularité des récits des patients. Pour défendre cette thèse, elle s'appuie sur des théoriciens littéraires et mentionne, entre autres, Gérard Genette dans son « Discours du récit » (1972) :

« Il n’y a pas de récit (ou de narration?) qui puisse être raconté en dehors de son énonciation. Dans Discours $d u$ récit, un commentaire sur $A ̀$ la recherche du temps perdu de Proust, Genette écrit : 'la spécificité de la narration proustienne prise dans son ensemble est irréductible, et toute extrapolation serait ici une faute de méthode; la Recherche n'illustre qu'elle-même”. » (Charon 2006, p. 45)

À la suite de cette référence, et voulant illustrer l'absolue singularité de chaque patient et de son expérience, Charon donne l'exemple d'un dermatologue diagnostiquant un psoriasis. Elle explique que celui-ci se fourvoierait s'il ne s'intéressait qu'à la catégorie universelle et médicale qu'il diagnostique, devant au contraire reconnaître le cas singulier devant lui : le psoriasis de son patient n'illustrerait que lui-même. Tout d'abord, cette interprétation de Genette est malheureuse. C'est notamment omettre la phrase qui suit et qui prend à rebours cette affirmation. Genette écrit ainsi :

« La Recherche n'illustre qu'elle-même. Mais, » ajoute-til, « d'un autre côté, cette spécificité n'est pas indécomposable, et chacun des traits qu'y dégage l'analyse se prête à quelque rapprochement, comparaison ou mise en perspective. Comme toute œuvre, comme tout organisme, la Recherche est faite d'éléments universels, ou du moins transindividuels, qu'elle assemble en une synthèse spécifique, en une totalité singulière. L'analyser, c'est aller non du général au particulier, mais bien du particulier au général. »

Lorsque Genette souligne l'absolue singularité de l'œuvre de Proust, ce n'est pas dans le but d'exclure la possibilité de toute réflexion d'ordre général sur celle-ci. Il est d'ailleurs intéressant de noter la comparaison opérée par Genette entre une œuvre et un organisme : un organisme, bien qu'absolument singulier et unique, partage de nombreux traits avec d'autres

5-Galen Strawson a notamment critiqué avec véhémence à la fois la version descriptive de la thèse de lidentité narrative (selon laquelle nous sommes véritablement nos récits) et la version normative de cette thèse (selon laquelle il est souhaitable de vivre sa vie comme un récit ou à travers des récits) (Strawson, 20o4).

6 - Implicitement, il faut donc comprendre aussi que la biomédecine, en tant qu'elle est une science, est considérée comme étant incapable de prendre en compte la singularité de l'expérience subjective (par cela, Charon entend souvent, l'expérience psychologique et émotionnelle) des patients. Pour revenir sur les limites de cette critique de la biomédecine, voir (Ferry-Danini, 2018).

7 - En français dans le texte, mais avec une coquille (« le narration »). 


\section{LA MÉDECINE NARRATIVE FACE À L'IMPOSSIBLE SINGULARITÉ DES RÉCITS}

et peut être ainsi étudié. Ce passage souligne bien que selon Genette, l'analyse et la critique littéraire n'ont pas vocation à être une connaissance pure du singulier et de l'unique. Une telle connaissance serait-elle même possible ? Si la totalité d'une œuvre ou d'un organisme est singulière, ses composés ne le sont pas. Comme le souligne Genette, si la Recherche est un récit singulier, elle comporte des ressemblances et des généralités communes à d'autres récits, s'imprègne et se tisse d'intertextualité. Elle n'est pas une œuvre « close » (Genette 1972, 67). Faisant à nouveau référence à Genette, Rita Charon poursuit et cite la fin de l'essai, dans lequel Genette écrit que « le général est au cour du singulier, et donc - contrairement au préjugé commun - le connaissable au cœur du mystère » (Genette, 1972, p. 69). De cette citation, Charon semble ne retenir qu'une idée, celle selon laquelle le singulier serait un mystère susceptible d'accès. Cette voie d'accès, elle la décrit - en empruntant ses mots à un travailleur social - comme une «transformation magique » et un « don » (« a gift ») (Charon, 2006, p. 48). Or cette idée - une sorte de connaissance intuitive du singulier qui serait possible lors de la rencontre «magique » avec autrui - en plus d'être simplement supposée et non défendue philosophiquement, va tout à fait à l'encontre de la narratologie et donc des racines mêmes de la médecine narrative. En effet, c'est bien la possibilité d'identifier des types de récits ou des types de procédés textuels qui a donné naissance à la médecine narrative. Le premier geste théorique d'un de ses précurseurs, Arthur Frank (1995), est notamment de proposer une classification sous la forme de trois types de schémas narratifs propres à la maladie : le schéma de la restitution, le schéma du chaos, et le schéma de la rédemption. Comme le note la philosophe Miriam Solomon, plusieurs tropes narratifs ou clichés peuvent être distingués, comme le récit de la gratitude envers les personnels soignants ou, dans le cas d'une narration plus épique, en forme de combat ou de quête, l'idée que chaque instant compte contre la maladie; ou bien encore, l'émergence d'une conscience politique (Solomon, 2015, p. 197) :

« L'espoir d'une restitution simple, par la suite anéanti, la descente aux enfers et le désespoir qui s'en suit, et finalement la rédemption portée par une quête où la vie de la personne s'en trouve enrichie. Les métaphores militaires de la bataille ou de la guerre contre une maladie sont communes » (Solomon, 2015, p.198)

De la même façon, le sociologue Mike Bury souligne que les récits de maladie - aussi singuliers qu'ils soient - s'insèrent dans un tissu culturel « qui propose des formes de langage spécifique, des clichés, des motifs, des références et d'autres éléments de répertoires linguistiques et symboliques qui permettent et contraignent ce qui est dit et comment » (Bury, 2001, p. 278) ${ }^{8}$. Si les récits ne sont pas clos, et qu'ils s'appuient sur un même tissu culturel et social, cela signifie aussi qu'ils ne sont pas absolument singuliers : ils comportent des ressemblances et des clichés communs à tous. Ils sont uniques comme peuvent l'être deux feuilles d'arbres similaires quoique singulières. L'inverse serait tout aussi incohérent qu'un langage privé (Wittgenstein, 2005, paras. 243-301) Si un récit était absolument singulier, il n'aurait pas de critères de compréhension indépendants de lui-même et ne pourrait être compris par personne. En insistant sur l'importance de la singularité des récits, la médecine narrative s'est irrémédiablement écartée de ses origines narratologiques. Cette tension entre la fondation narratologique de la médecine narrative et l'importance cruciale qu'elle attribue à la singularité des expériences et des récits, demeure non résolue.

Cette tension s'exprime par exemple dans l'anonymisation des récits que raconte Rita Charon. Chaque récit est ainsi anonymisé pour qu'une personne particulière ne soit pas reconnue. Comme le remarque Miriam Solomon, cela signifie que chaque histoire est racontée comme appartenant à un type d'histoires (Solomon, 2015, p. 197), ce qui laisse supposer que ce qui importe pour la médecine narrative n'est pas la singularité absolue de chaque histoire. Ce procédé d'anonymisation se retrouve chez la plupart des défenseurs de la médecine narrative. Il est ainsi étonnant que pour Charon, le récit de la maladie par le patient soit conçu comme le siège de la singularité - et de la subjectivité. Elle écrit même que c'est par « cette sphère de la narration que les patients ont enfin pu essayer de reprendre main sur leur singularité, leur subjectivité. (...) », allant même jusqu'à affirmer que « la nouvelle autorité des patients sur leur récit de maladie », «prouve la thèse selon laquelle la singularité réside dans la narration » (Charon, 2006, p. 47). Cette tension entre une thèse absolument singulariste et une approche narratologique n'est pas expliquée.

Dans ce qui suit, je propose de m'intéresser aux conséquences de ce problème, qui dépassent le cadre théorique de la médecine narrative. La thèse de l'unicité des récits des patients n'entre pas simplement en tension avec l'étude des récits des patients - et donc la médecine narrative - elle peut aussi tendre à masquer un ensemble de processus de domination et de régulation des récits. Pour développer ce point, je m'appuie sur une analyse des récits du cancer du sein aux ÉtatsUnis au XXe siècle proposée par Miriam Solomon (2015). Cet exemple illustre de façon frappante que les narrations de maladie ne sont pas absolument singulières, elles s'inscrivent dans un contexte social et culturel, et leur expression peut être régulée et dominée par un certain type de récit.

\section{4 - Quand des types de récits de maladie deviennent domi- nants : l'exemple du cancer du sein aux États-Unis au XXe siècle}

Le récit type du cancer du sein aux États-Unis au XXe siècle appartient à un genre bien particulier de récit de maladie. Dans la classification proposée par Ian Robinson (1990), on parlerait de narration " progressive », c'est-à-dire d'une narration où la patiente reconstruit sous un jour positif les événements négatifs liés à sa maladie. Dans la taxonomie proposée par Bury (2001), on parlerait de narration de type « moral », narration au statut particulièrement ambigu. Dans ce type de 


\section{LA MÉDECINE NARRATIVE FACE À L'IMPOSSIBLE SINGULARITÉ DES RÉCITS}

récit, on retrouve la volonté de la patiente de se donner une apparence vertueuse dans la maladie, de ne pas « se laisser aller », comme l'analyse Bury. Ces types de récits sont parfois décrits comme allant souvent de pair avec l'expression d'une culture de l'épanouissement ou de développement personnel, particulièrement prégnante dans la culture américaine (Bury, 2001, p. 276).

Voici comment Miriam Solomon décrit le récit type d'un cancer du sein aux États-Unis : survient d'abord la découverte d'une masse suspecte lors d'un examen; cette découverte est suivie d'un sentiment d'inquiétude et d'espoir que cette masse ne se révèle pas pathologique, puis par le désespoir lorsque le diagnostic du cancer est confirmé. Caractéristique d'un récit de type " progressif » ou « moral », vient ensuite l'expression d'un but nouveau : cette raison d'être renouvelée permet à la malade de venir à bout des épreuves que sont les traitements, jusqu'à la transformation positive de sa personnalité (Solomon, 2015, p. 198). Le récit autobiographique de Betty Rollin, « D'abord, vous pleurez... » (1976) en est l'une des premières illustrations aux États-Unis. La quatrième de couverture annonce "l'histoire vraie d'une femme qui a transformé l'épreuve la plus terrifiante de sa vie en un nouveau départ ». Depuis Rollin, on assiste à une médiatisation du cancer du sein, les autobiographies et les récits de fiction sur le sujet se multipliant. Benoît Lafon remarque ainsi que le nombre de fictions sur le cancer en général, genre introduit dans les années 1970 à la télévision, n'a cessé de croître (Lafon, 2007), le portrait de femme atteinte par le cancer devenant un genre médiatique majeur'. Comme l'écrit l'activiste Ehrenreich, le cancer du sein est « la plus importante maladie de la carte culturelle, plus importante que le sida, la mucoviscidose ou les blessures vertébrales, plus importante encore que ces maladies qui tuent le plus de femmes - maladies du cœur, cancer du poumon, crises cardiaques » (Ehrenreich, 2001, p. 45). Le récit des cancers est ainsi paradoxalement plus médiatisé que les récits d'autres maladies de gravité et d’incidence similaires, voire supérieures.

Les études féministes se sont particulièrement intéressées à cette médiatisation accrue des récits de cancer féminin. À la suite de l'analyse de Solomon, on peut mentionner les travaux de Judy Segal (2008; 2015) et de Barbara Ehrenreich (2001; 2010). Activiste politique, auteure et journaliste, Barbara Ehrenreich a fait l'expérience de ce récit type de cancer du sein lors de son diagnostic et de son traitement. Elle décrit cette expérience comme la rencontre avec un univers ou un " monde » du cancer du sein aux États-Unis, une culture « joyeuse », écrit-elle, où l'on porte avec bravoure un ruban rose sur le col de son manteau, et qu'elle critique à plusieurs reprises dans ses articles ironiquement intitulés « Souriez! Vous avez le cancer ! » (2010) et «Bienvenue à Cancerland : d'une mammographie au culte du kitsch rose » (2001). Elle décrit ainsi l'optimisme qui prime dans cette culture - ou culte, selon ses mots. Les malades qui guérissent sont ainsi communément désignées sous le terme de "survivantes » (« survivors »), mieux traduit en français par « battantes» ou « combattantes », ce qui correspond au trope commun de la bataille et de la victoire contre la maladie. Ce monde du cancer du sein se traduit aussi par une médiatisation faite d'opérations soutenues par de grands groupes privés, où des peluches et des rubans roses sont distribués : l'image morbide de la maladie est ainsi occultée (Ehrenreich, 2001, p. 48). Ehrenreich décrit une atmosphère incitant à une « adhésion positive à la maladie » (Ehrenreich, 2001, p. 48); pour le dire autrement, une atmosphère où les narrations exprimant la colère, la détresse ou la négativité face au cancer du sein n'ont pas leur place. À ce propos, Ehrenreich relate une anecdote significative. Recherchant une forme de soutien sur un forum d'entraide de patientes atteintes du cancer du sein, elle poste un message, où sous le titre " En colère », elle décrit ses doléances à propos de sa maladie, son traitement et le système médical. Dans les réponses à son poste, Ehrenreich est critiquée pour son « mauvais esprit » et sa négativité, qui ne pourraient, selon ses interlocutrices, que diminuer ses chances de guérir. Une voix dissidente s'est pourtant fait entendre, celle d'une patiente, Gerri, en stade terminal, qui explique avoir « tout bien fait » pour guérir; le cancer n'est pas rien, «IT IS NOT O.K! » (2001, p. 50), s'exprime-t-elle avec colère.

Au-delà du cas du cancer du sein, on retrouve ce type de narration « morale » (Bury, 2001) ou " progressive » (Robinson, 1990) dans le monde des maladies chroniques aux ÉtatsUnis, mais pas uniquement. On retrouve ainsi la critique de cet impératif d' " accueil positif de la maladie » sous la plume de Ruwen Ogien, dans son dernier livre paru peu après sa mort, « Mes mille et une nuits » (Ogien, 2017). Il désigne cet impératif sous le terme de dolorisme, l'idée qu'il faille absolument trouver du sens dans la souffrance. Il est intéressant de remarquer que la médecine narrative n’a jamais donné écho à ce genre de critiques. Ainsi, chez Arthur Frank, on trouve à l'inverse l'expression et la revendication explicite de ce type d'approche morale ou de dolorisme. Dans la maladie chronique, selon lui, les patients espèrent devenir " victorieusement malades » (« successfully ill ») (Frank, 1997, p. 117) ${ }^{10}$. Il conçoit la maladie et la souffrance comme l'occasion « d'une ouverture morale pour témoigner et changer » (Frank, 1997, p. 141). Il ne s'agit plus seulement de la réussite d'un traitement, mais bien d'être malade avec réussite. Selon Bury, cet état d'esprit témoigne d'un fort ancrage religieux et chrétien, où la rédemption se trouve via la souffrance ${ }^{11}$. Selon ses mots,

«L'individu en souffrance et en détresse est souvent décrit en des termes romantiques sinon sentimentaux, et les relations réelles dans lesquelles est engagé l'individu peuvent être perdues dans l'importance excessive donnée aux « narrations personnelles » positives qui sont reproduites sans distance critique par les sociologues. » (Bury, 2001, p. 277)

La narration typique du cancer du sein, dominant l'espace médiatique et narratif, introduit une norme de conduites à adopter : elle ne permet pas aux malades de dévier de cette 
REVUE

DE LA SOCIÉTÉ

DE PHILOSOPHIE

DES SCIENCES

\section{LA MÉDECINE NARRATIVE FACE À L'IMPOSSIBLE SINGULARITÉ DES RÉCITS}

narration optimiste. En effet, il est souvent admis que l'une des fonctions de la narration, autobiographique ou fictive, est de proposer des rôles sociaux aux malades. C'est en tout cas la position d'auteurs comme Schaeffer (1999), dans le cas de la fiction, qui selon lui peut être la base d'un processus de faire semblant du rôle de la patiente. L'existence de narration dominante " régule », selon les mots de Judy Segal (2008), les rôles narratifs et sociaux disponibles. Certains rôles sont valorisés, d'autres dévalorisés et certains tout à fait absents.

\section{5 - Conclusion}

La médecine narrative dans sa version la plus répandue fait ainsi face à plusieurs limites. La thèse de la singularité des récits des patients entre en effet en tension avec l'édifice argumentatif de l'approche, qui repose sur la tradition narratologique à laquelle la médecine narrative emprunte le nom. Bien que l'on puisse souligner l'unicité certes réelle des récits des patients, il ne semble pas que 1) cette unicité de principe soit utile d'un point de vue narratologique. Au contraire, il semble que l'intérêt d'une approche narrative découle précisément des ressemblances entre les récits des patients; 2) d'autre part, ce présupposé tend à occulter certains effets régulateurs, menant à une uniformisation des récits, et qui peuvent empêcher une patiente de proposer un récit original.

Cette tension au cœur de la médecine narrative peut cependant facilement être dépassée, puisqu'il suffit, pour y répondre, de revenir aux racines narratologiques de l'approche, afin de prendre en compte les ressemblances entre les récits des patients et non pas simplement leur singularité absolue. Cependant, il fallait souligner que ces ressemblances sont certes utiles d'un point de vue de l'analyse narratologique, mais qu'elles peuvent cacher ou exprimer une certaine uniformisation des récits. Enfin, il faut signaler les travaux en narratologie qui vont tout à fait à l'encontre du présupposé de la médecine narrative selon lequel il est toujours possible et souhaitable de raconter sa maladie. Rimmon-Kenan notamment (Rimmon-Kenan, 2002; 2006) a souligné que l'expérience de la maladie peut être un chaos tel qu'il devient impossible d'en proposer une narration. Selon elle, les outils narratologiques ne sont pas toujours capables de formuler un récit narratif et de produire une narration « morale » ou " progressive ». Selon elle, cette incapacité n'est pas à analyser comme un échec imputable aux personnes malades, mais comme étant constitutive de l'expérience chaotique de la maladie.

\section{RÉFÉRENCES}

BRODY, Howard. 1997. Who Gets to Tell the Story? Narrative in Postmodern Bioethics. In LINDEMANN, Hilde (dir.). Stories and Their Limits: Narrative Approaches to Bioethics. New York: Routledge. 18-30.

BURY, Mike. 2001. llness narratives: fact or fiction?. Sociology of Health \& Illness, 23 (3), 263-285. Lien

CHARON, Rita. 2001. Narrative Medicine: A Model for Empathy, Reflection, Profession, and Trust, Jama, 286 (15), 1897-1902. Lien

CHARON, Rita. 2006. Narrative Medicine: Honoring the Stories of Illness. Oxford and New York: Oxford University Press.

EHRENREICH, Barbara. 2001. Welcome to cancerland. Harper's Magazine. November. 43-53.

EHRENREICH, Barbara. 2010. Smile! You've got cancer. The Guardian, January 2, section Society.

FERRY-DANINI, Juliette. 2018. A New Path for Humanistic Medicine. Theoretical Medicine and Bioethics, 39 (1), 57-77. $\underline{\text { Lien }}$

FRANK, Arthur W. 1995. The Wounded Storyteller: Body, Illness, and Ethics. $1^{\text {er }}$ éd. Chicago: University of Chicago Press. Lien

FRANK, Arthur W. 1997. Enacting Illness Stories: When, What, and Why. In LINDEMANN, Hilde (dir.). Stories and Their Limits: Narrative Approaches to Bioethics. New York: Routledge, 31-49.

GENETTE, Gérard. 1972. Figures III. Paris : Seuil.

GOUPY, François, LE JEUNE, Claire. 2016. La médecine narrative : Une révolution pédagogique?, Paris : Editions Med Line, 262.

HUNTER, Kathryn Montgomery. 1991. Doctors'Stories: The Narrative Structure of Medical Knowledge. Princeton: Princeton University Press.

LAFON, Benoît. 2007. Le cancer en prime time. Émergence d'une confrontation distanciée avec la maladie par les fictions télévisées. Questions de communication. 11, 129-148. Lien OGIEN, Ruwen. 2017. Mes mille et une nuits - La maladie comme drame et comme comédie. Albin Michel.

O’MAHONY, Seamus. 2013. Against Narrative Medicine. Perspectives in Biology and Medicine, 56 (4), 611-619. Lien REISS, Julian, SOLOMON, Miriam, TEIRA, David. 2011. Mechanisms, continental approaches, trials, and evolutionary medicine: New work in the philosophy of medicine. Theoretical Medicine and Bioethics, 32 (1), 1-4. Lien

RIMMON-KENAN, Shlomith. 2002. The Story of "I": Illness and Narrative Identity ». Narrative, 10 (1), 9-27. Lien RIMMON-KENAN, Shlomith. 2006. What Can Narrative Theory Learn from Illness Narratives?. Literature and Medicine, 25 (2), 241-254. Lien

ROBINSON, Ian. 1990. Personal Narratives, Social Careers and Medical Courses: Analysing life trajectories in autobiographies of people with multiple sclerosis. Social Science \& Medicine (1982), 30 (11), 1173-1186. Lien

ROLLIN, Betty. 1988. D'abord vous pleurez.... Traduit par Marie-Jo Demoulin-Astre. Paris : P. Belfond.

SCHAEFFER, Jean-Marie. 1999. Pourquoi la fiction?, Paris : Seuil.

SEGAL, Judy Z. 2008. Breast Cancer Narratives as Public 
Rhetoric: Genre Itself and the Maintenance of Igorance. Linguistics and the Human Sciences, 3 (1), 3-23. Lien

SEGAL, Judy Z. 2015. The View from Here and There: Objectivity and the Rhetoric of Breast Cancer. In PADOVANI, Flavia, RICHARDSON, Alan, TSOU, Jonathan (eds). Objectivity in Science. Boston Studies in the Philosophy and History of Science, 310. Cham: Springer. 211-226. Lien

SOLOMON, Miriam. 2008. Epistemological Reflections on the Art of Medicine and Narrative Medicine. Perspectives in Biology and Medicine, 51 (3), 406-417. Lien

SOLOMON, Miriam. 2015. Making Medical Knowledge. Oxford: Oxford University Press. Lien

STRAWSON, Galen. 2004, Against Narrativity ». Ratio, 17 (4), 428-452. Lien

VANNATTA, Seth, VANNATTA, Jerry. 2013. Functional Realism: A Defense of Narrative Medicine. The Journal of Medicine and Philosophy: A Forum for Bioethics and Philosophy of Medicine, 38 (1), 32-49. Lien

WITTGENSTEIN, Ludwig. 2005. Recherches philosophiques. Traduit par Françoise Dastur, Maurice Elie, JeanLuc Gautero, Dominique Janicaud, Elisabeth Rigal. Paris : Gallimard, coll. Bibliothèque de philosophie.

WOODS, Angela. 2011. The limits of narrative: provocations for the medical humanities. Medical Humanities, 37 (2), 73-78. Lien

HISTORIQUE

Article initialement soumis le 15 octobre 2019

Article révisé soumis le 6 février 2020

Article accepté le 7 février 2020.

SITE WEB DE LA REVUE

ojs.uclouvain.be/index.php/latosensu

ISSN 2295-8029

DOI http://dx.doi.org/10.20416/LSRSPS.V7I2.1

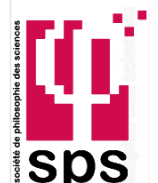

SOCIÉTÉ DE PHILOSOPHIE DES SCIENCES (SPS) École normale supérieure 45, rue d'Ulm 75005 Paris www.sps-philoscience.org
CONTACT ET COORDONNÉES :

Juliette Ferry-Danini

1, rue Victor Cousin 75005 Paris

ferry.danini@gmail.com 